\title{
Histological long-term effects in relevant tissues after 131 l contamination of two Wistar rat models
}

\author{
Meriem Mezaguer-Lekouaghet ${ }^{1, *}$, Abdelwahab Badreddine ${ }^{1}$, Saadia Mameri ${ }^{2}$, Maamar \\ Souidi ${ }^{3}$, Ahcène Baz ${ }^{4}$, and Zohra Lounis-Mokrani ${ }^{1}$ \\ ${ }^{1}$ Centre de Recherche Nucléaire d'Alger, Algérie \\ ${ }^{2}$ Centre Hospitalo Universitaire Mustapha Bacha Alger, Algérie \\ ${ }^{3}$ Institut de Radioprotection et de Sûreté Nucléaire Paris, France \\ ${ }^{4}$ Ecole Normale Supérieure de Kouba, Algérie
}

\begin{abstract}
Procedures using iodine-131 $\left({ }^{131} \mathrm{I}\right)$ represent about $90 \%$ of all therapies in nuclear medicine $[1,2]$. It is important to evaluate the long-term biological effects of ${ }^{131}$ I treatment on nontarget organs in order to improve the patient radioprotection.

The aim of this work is to use an experimental animal model to contribute to the understanding of some biological effects induced by ${ }^{131} \mathrm{I}$ contamination, and especially histological effects.

Two models of Wistar rats with $(\operatorname{Tr}+)$ and without thyroid $(\operatorname{Tr}-)$ were orally contaminated with iodine and after 8 months they were sacrificed and the histological effects on some relevant tissues such as thyroid, testes, liver and kidneys were been studied.

Thyroid tissue analysis revealed that in the case of the $\mathrm{Tr}+$ model compared to their control ( $\mathrm{Tr}+$ uncontaminated), 50\% of the slides examined had remodeled the thyroid tissue with rare follicles choked by fibrosis and with epithelial changes. However, for Trcompared to their control, the examined slides reveal the presence of a small, completely atrophied thyroid mass associated with vesicular fibrosis and with detachment of the colloid.

For the renal organ, disturbances are observed: inflammation of the presence of tissue fibrosis and glomerular necrosis. For the liver, there is an appearance of inflammatory focus in different degrees around the portals. However, the results of the testes of both models compared to their controls revealed no histological abnormalities. The observed histological effects are correlated with the corresponding absorbed dose received by each organ and calculated using the RODES software $[3,4]$.
\end{abstract}

\section{References}

[1]. International Commission on Radiological Protection.. Oxford: Pergamon Press; ICRP Publication 94 (2004).

\footnotetext{
${ }^{\star}$ Corresponding author: m_mezaguer@yahoo.fr
} 
[2]. United Nations Scientific Committee on the Effects of Atomic Radiation. Sources and effects of ionizing radiation. 2000 report to the general assembly with annexes. Vienna: United Nations; 2001. [3]. M. Mezaguer-Lekouaghet, A. Badreddine, N. Ait Kaci, K. Remil, A. Benbournane, F. Boucheneb, F. Messili, A. Baz, M. Souidi, \& Z. Lounis-Mokrani, ICNTRM. Strasbourg France (2017).

[4]. M. Mezaguer-Lekouaghet, Eric Blanchardon, A. Badreddine, Jean-Marc Bertho, Maamar Souidi, Ahcène Baz \& Z. Lounis-Mokrani. (It is presented in the same conference). 